\title{
Hsa_circ_0001017 inhibits proliferation and metastasis via regulating the let-7g-3p/NDST3 axis in glioma
}

\author{
Tao $\mathrm{Li}^{1 \#}$, Yunlong $\mathrm{Li}^{2 \#}$, Wei Zhang ${ }^{3}$, Jing Zhang ${ }^{4}$ \\ ${ }^{1}$ Department of Neurosurgery, Shengli Oilfield Central Hospital, Dongying, Shandong, P.R. China, ${ }^{2}$ Department of Neurosurgery, \\ Weifang Yidu Central Hospital, Qingzhou, Shandong, P.R. China, ${ }^{3}$ Department of Neurosurgery, Chengyang District People's Hospital, \\ Qingdao, Shandong, P.R. China, ${ }^{4}$ Department of Internal Medicine-Neurology, The First Affiliated Hospital of Shandong First Medical \\ University, Jinan Shandong, P.R. China \\ \#Tao Li and Yunlong Li contributed equally to this work.
}

\begin{abstract}
Introduction: Glioma is one of the common types of intracranial malignancies, which seriously threatens human health. Circular RNAs (circRNAs) play critical roles in various tumours. This study aims to observe the functions and mechanism of circ_0001017 in glioma progression.

Material and methods: Hsa_circ 0001017 expression was analysed in glioma tissue and cells using qRT-PCR assay. Flow cytometer, Cell Counting Kit-8 (CCK-8), colony formation, wound healing, and Transwell were used to analyse glioma cell apoptosis, viability, colony-forming ability, migration, and invasion. The expression of $\mathrm{N}$-deacetylase and $\mathrm{N}$-sulfotransferase 3 (NDST3) and epithelial-mesenchymal transition (EMT)-related proteins was measured using western blot analysis. Luciferase reporter and RNA immunoprecipitation (RIP) assay were performed to determine the target relationship.

Results: In glioma tissues and cells, hsa_circ_0001017 expression was decreased. The overexpression of hsa_ circ 0001017 inhibited glioma cell proliferation, EMT, migration, and invasion and promoted glioma cell apoptosis, while the knockdown of hsa_circ_0001017 caused the opposite results. Mechanistically, hsa_circ_0001017 sponged hsa-let-7g-3p and NDST3 was the target gene of hsa-let-7g-3p. Moreover, the tumour-suppressive role of circ_0001017 was associated with hsa-let-7g-3p and NDST3.

Conclusions: Hsa_circ_0001017 suppressed the growth and metastasis and increased cell apoptosis of glioma, and this effect was associated with hsa-let-7g-3p/NDST3 axis.
\end{abstract}

Key words: hsa_circ_0001017, hsa-let-7g-3p, NDST3, glioma.

\section{Introduction}

Glioma is a common intracranial malignancy in adults, which has a high mortality rate [4,31]. Currently, multimodal treatments, such as radical sur- gery and chemo-radiotherapy, have been improved, but the incidence rate of glioma continues to increase and the prognosis of glioma remains poor $[22,40]$. The poor therapeutic effect is mainly because many

Communicating author:

Dr. Jing Zhang, Department of Internal Medicine-Neurology, The First Affiliated Hospital of Shandong First Medical University,

No. 16766, Jingshi Road, Jinan, Shandong, 250014, P.R. China, e-mail: jingzhang789@126.com 
patients suffer from advanced and metastatic disease [38]. Thus, the potential mechanisms of glioma need to be explored, and novel and effective therapeutic targets for glioma need to be determined.

Circular RNAs (circRNAs), expressed in highly divergent eukaryotes, are endogenous noncoding RNAs (ncRNAs) [27,30]. CircRNAs have no 5' cap and 3 ' ploy (A) tail, but have a covalently closed loop structure; thus, they can maintain high cellular stability $[12,21]$. CircRNAs are considered to be novel plasma biomarkers [17]. Moreover, circRNAs play an important part in various types of cancer, and the abnormal function of circRNAs affects the biological process of tumour cells $[8,9,19]$. Interestingly, many circRNAs have been found to be upregulated or downregulated in glioma. For example, Wang et al. [33] found that hsa_circ_0001649 expression decreased in glioma tissues and cells, and cell growth was inhibited by the overexpression of hsa_circ_0001649. Therefore, the role of circRNAs as novel biomarkers or therapeutic targets in glioma needs to be examined.

Emerging evidence shows that circRNA can serve as a competing endogenous RNA (ceRNA) in binding to microRNAs (miRNAs) to modulate gene expression $[25,36]$. MiRNAs are 22-nucleotide-long ncRNAs identified to take part in tumour progression [39]. A previous paper showed that circ_0034642 could increase BATF3 expression through miR 1205 to facilitate glioma cell proliferation and invasion [37]. Another paper [16] reported that hsa_circ_0046701 could promote carcinogenesis of glioma, and this effect was associated with the miR-142-3p/ITGB8 axis. However, the role of circRNA in glioma is under investigation.

Hsa_circ_0001017 was downregulated in dysplasia tissues and cancer, and the hsa_circ_0001017 expression levels were significantly related to distant metastasis [18]. However, the function of hsa_circ_0001017 in glioma is not fully clarified. In the present study, hsa_circ_0001017 expression in glioma tissues and cells was analysed. Moreover, the function of hsa_circ_0001017 and its potential molecular pathway in glioma were explored. The findings might offer a new insight into circRNAdirected treatment in glioma.

\section{Material and methods}

\section{Patients and tissue samples}

In this study, tumour tissue samples were obtained from 40 patients diagnosed with glioma in our hos- pital between January 2019 and June 2020. Normal brain tissues were obtained from 20 patients without craniocerebral injuries (cerebral haemorrhage, cerebral contusion and laceration). All registered patients did not receive preoperative radiotherapy and chemotherapy before surgery, and they signed informed consent. The research was authorised by the Ethics Committee of our hospital.

\section{Cell culture}

Human normal astrocytes (NHA) and glioma cells (U118, U251, U87MG and LN229) were purchased from the Chinese Academy of Sciences (Shanghai, China). All cells were cultured in DMEM medium containing $10 \%$ foetal bovine serum (FBS; Gibco, CA, USA) and penicillin/streptomycin at $37^{\circ} \mathrm{C}$ and $5 \% \mathrm{CO}_{2}$.

\section{Cell transfection}

U87MG cells were grouped into control (without treatment), si-NC (cells transfected with siRNA negativecontrol), si1-circ(cellstransfected withsiRNA1circ-0001017) and si2-circ group (cells transfected with siRNA2-circ-0001017) groups. U251 cells were divided into control (without treatment), vector (cells transfected with pcDNA3.1-NC), over-circ (cells transfected with pcDNA3.1-circ-0001017), let-7g-3p mimic (cells transfected with let-7g-3p mimic), over-circ + let-7g-3p mimic (cells transfected with let-7g-3p mimic and pcDNA3.1-circ-0001017) and let-7g-3p $+\mathrm{N}$-deacetylase and N-sulfotransferase (NDST3) (cells transfected with let-7g-3p mimic and pcDNA3.1-NDST3) groups. Negative control, circ-0001017 siRNA, pcDNA3.1-circ-0001017, pcDNA3.1-NDST3, and let-7g-3p mimic were purchased from GenePharma (Shanghai, China). Cell transfection was performed using Lipofectamine 3000 transfection reagent (Invitrogen, CA, USA).

\section{Quantitative reverse transcription polymerase chain reaction}

Total RNA was extracted using TRIzol reagent (Invitrogen) from tissue and cells. Complementary deoxyribonucleic acid (cDNA) was synthesized using a PrimeScript Reverse Transcription (RT) Reagent Kit with a gDNA Eraser (Takara, Shiga, Japan). In line with the instructions of SYBR $^{\circledR}$ Premix Ex Taq ${ }^{\mathrm{TM}}$ ॥ (Takara), mRNA expression was measured by quantitative RT-polymerase chain reaction (qRT-PCR) and 
Table I. The sequences of primers

\begin{tabular}{|lc|}
\hline Primers & Sequences (5'-3') \\
\hline circ-0001017-F & AACCAGTGCGAAGTAATCTATGC \\
\hline circ-0001017-R & TCTTTGCTGGGCTCCTTCT \\
\hline let-7g-3p-F & TGGTACTGATGTGATGGACT \\
\hline let-7g-3p-R & TCATATCACACAGCACCGAT \\
\hline NDST3-F & GCCCCTCCCAGGAGTAAGTA \\
\hline NDST3-R & AGGTGGTGGAGAGAACAGGT \\
\hline GAPDH-F & GGTCGGAGTCAACGGATTT \\
\hline GAPDH-R & TACCAGAGTTAAAAGCAGCC \\
\hline
\end{tabular}

normalized to GAPDH. The $2^{-\Delta \Delta C t}$ method was used to analyse the data. The primer sequences were shown in Table I.

\section{Cell viability assay}

After culturing for $0,24,48$, and $72 \mathrm{~h}$, the transfected cells were incubated with Cell Counting Kit-8 (CCK-8) solution (Beyotime Institute of Biotechnology, China). After incubation at $37^{\circ} \mathrm{C}$ for $2 \mathrm{~h}$, the optical density (OD) value was measured using a microplate reader (MultiScan FC, Thermo Scientific, NY, USA) at $450 \mathrm{~nm}$.

\section{Colony formation assay}

The transfected cells (1000 cells/well) were seeded into a 6-well plate. After 12 days of incubation, the cells were fixed with methanol (Sigma-Aldrich, Merck, Darmstadt, Germany) for 20 min and stained with crystal violet solution (Sigma-Aldrich) for $15 \mathrm{~min}$. Subsequently, the colonies were imaged and counted.

\section{Cell apoptosis assay}

The cells were collected after transfection for $48 \mathrm{~h}$ and re-suspended in $400 \mu$ of $1 \times$ binding buffer. Next, the cells were incubated with Annexin V-FITC $(5 \mu \mathrm{l})$ and propidium iodide (PI, $5 \mu \mathrm{l}$; BD Biosciences, NJ, USA) in the dark for 15 min. Finally, flow cytometer analysis (BD Biosciences) was used to detect the cell apoptosis.

\section{Wound healing assay}

The transfected cells $\left(5 \times 10^{5}\right.$ cells/well) were seeded into a 6 -well plate and maintained in serumfree medium. A 200- $\mu$ l pipette tip was used to create a straight scratch (time $0 \mathrm{~h}$ ) when the cell confluence reached about $90 \%$ confluence. After scratch for 0 and $48 \mathrm{~h}$, the images were captured under a microscope (Olympus, Japan).

\section{Transwell assay}

The 24-well Transwell chamber (BD Biosciences) with membranes having $8-\mathrm{mm}$ pores was used to detect cell invasion. In short, $5 \times 10^{4}$ cells/well transfected cells were added into the Matrigel-coated upper chamber containing $500 \mu$ of serum-free medium. DMEM medium with $10 \%$ FBS was added to the lower chamber. The cells in the lower chamber were fixed with methanol for 10 min and stained with $0.1 \%$ crystal violet after incubation for $48 \mathrm{~h}$. Finally, under a microscope, the invasive cells were counted and imaged.

\section{Western blot analysis}

RIPA lysis buffer (CWBio, Beijing, China) with protease inhibitors was performed to extract total proteins. Equal amounts of protein sample was loaded into SDS-PAGE to fractionate the extracted proteins, and then transferred to PVDF membranes (Merck Millipore, Burlington, MA). Blocking with $5 \%$ non-fat milk for $1 \mathrm{~h}$ at $37^{\circ} \mathrm{C}$, the membranes were incubated with primary antibodies (Vimentin, 5741; N-cadherin, 13116; E-cadherin, 3195; GAPDH, 5174, 1 : 1000; Cell Signaling Technologies, Danvers, MA, USA; NDST3, SAB2101559, 1 : 1000; Sigma-Aldrich) at $4^{\circ} \mathrm{C}$ overnight. Next, the membranes were incubated with HRP-labelled secondary antibody (1 : 5000, Cell Signaling Technologies) at room temperature for $1 \mathrm{~h}$. Finally, the enhanced chemiluminescence (ECL) system (Thermo Scientific, USA) was used to visualize the bands.

\section{RNA immunoprecipitation assay}

U251 cells were lysed in complete RNA immunoprecipitation (RIP) lysis buffer (Merck Millipore). Next, the cell lysates were hatched with RIP buffer containing magnetic beads bound to a human anti-Argonaute2 (Ago2) antibody (Merck Millipore) or immunoglobulin G (IgG, Merck Millipore). Proteinase K was incubated with the samples, and then immunoprecipitated RNA was isolated for detecting the levels of hsa_circ_0001017, hsa-let-7g-3p, and NDST3. 


\section{Luciferase reporter assay}

GeneChem Co., Ltd (Shanghai, China) constructed the luciferase reporter plasmid (hsa_circ_0001017WT, hsa_circ_0001017-MUT, NDST3-WT and NDST3MUT). U251 cells were co-transfected with corresponding plasmids and let-7g-3p mimics or its negative control using Lipofectamine 3000 transfection reagent. The luciferase activity was measured using Dual-Luciferase Reporter Assay System (Promega, WI, USA) after transfection for $48 \mathrm{~h}$.

\section{Statistical analysis}

Statistical analysis was performed by GraphPad Prism 7 (GraphPad Software, Inc., La Jolla, CA, USA). Data were shown as mean \pm standard deviation (SD) from three independent experiments. The Student $t$ test and one-way ANOVA were used to analyse the difference. $P<0.05$ was considered to be statistically significant.

\section{Results}

\section{Hsa_circ_0001017 expression decreases in glioma tissues and cells}

The hsa_circ_0001017 expression in glioma tissue and cells was detected using a qRT-PCR assay to assess the role of hsa_circ_0001017 in glioma. The hsa_circ_0001017 expression was decreased in glioma tissues compared with non-neoplastic brain tissues (Fig. 1A, $p$ < 0.01). Furthermore, compared with the NHA cells, the hsa_circ_0001017 expression was decreased in glioma cells (U251, U118, LN229, and U87MG) (Fig. 1B, $p<0.01$ ).

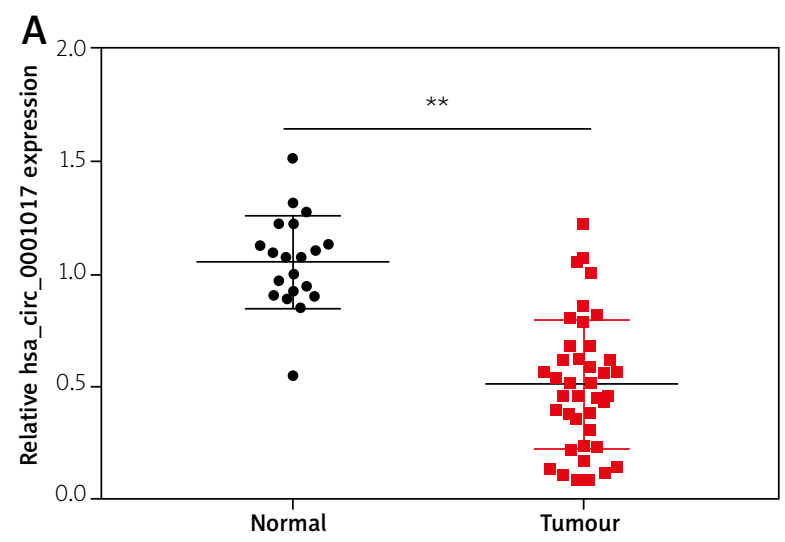

\section{Hsa_circ_0001017 restrains glioma cell proliferation and induces glioma cell apoptosis}

U251 cells with the lowest hsa_circ_0001017 expression and U87MG cells with the highest hsa_ circ_0001017 expression were used for gain or lossof-function experiments to illustrate the function of hsa_circ_0001017 in glioma. Hsa_circ_0001017 was stably overexpressed or knocked down in glioma cells (Fig. 2A). According to Figure 2B-D, the overexpressed hsa_circ_0001017 restrained the proliferation ability and increased the apoptosis rate of U251 cells ( $p<0.05$ ), while the knockdown of hsa_circ_0001017 increased the proliferation ability and decreased the apoptosis rate of U87MG cells $(p<0.05)$.

\section{Hsa_circ_0001017 restrains glioma cell migration, invasion, and epithelial- mesenchymal transition}

Wound healing and Transwell assays were performed to assess the influence of hsa_circ_0001017 on cell metastatic property. The over-expressed hsa_ circ_0001017 restrained the migration capacity of U251 cells, but the knockdown of hsa_circ_0001017 promoted the migration capacity of U87MG cells ( $p<0.01$, Fig. 3A). Simultaneously, the Transwell invasion assay also showed a similar result (Fig. 3B). The epithelial-mesenchymal transition (EMT)-related proteins expression was examined using western blot analysis to clarify further the mechanism of action of hsa_circ_0001017 in cell metastatic

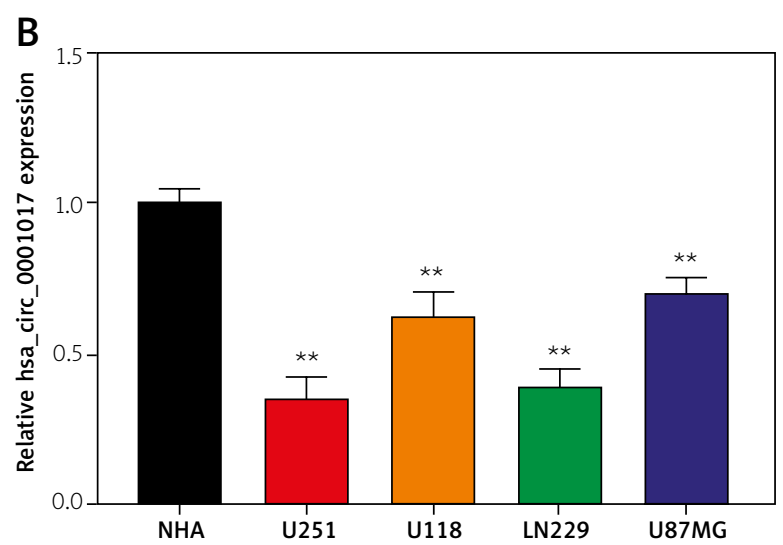

Fig. 1. Hsa_circ_0001017 expression was decreased in glioma tissues and cells. A) The hsa_circ_0001017 expression in glioma tissue $(n=40)$ and non-neoplastic brain tissues (normal, $n=20$ ) was measured using qRT-PCR assay. B) The hsa_circ_0001017 expression in human normal astrocytes (NHA) and glioma cells (U118, U251, U87MG and LN229) was detected using qRT-PCR assay. ${ }^{* *} p<0.01$ vs. normal group or NHA cells. 

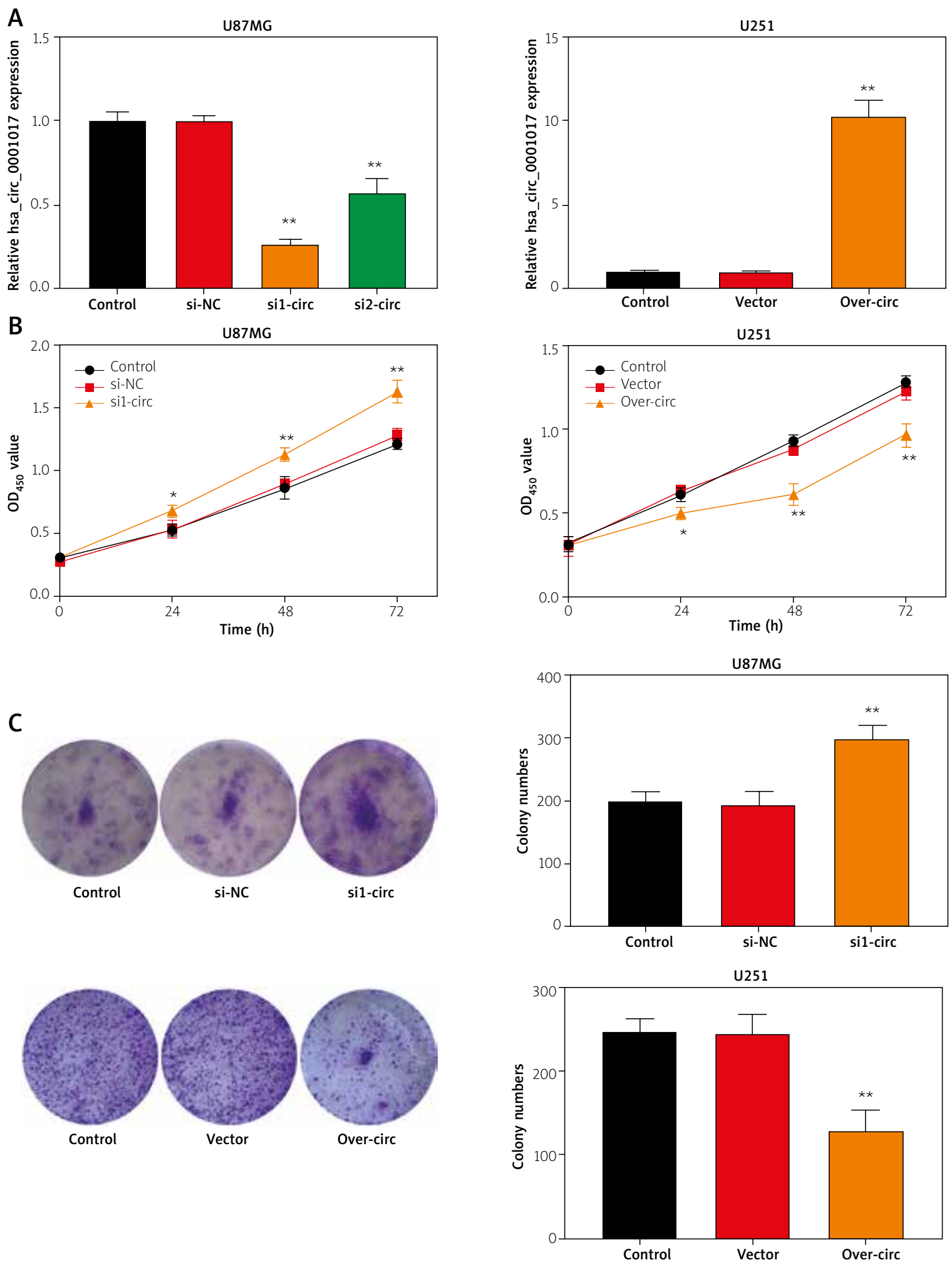

Fig. 2. Hsa_circ_0001017 inhibited glioma cell proliferation and promoted glioma cell apoptosis. A) The hsa_circ_0001017 expression in U87MG and U251 cells was analysed through GRT-PCR assay. B) The cell viability of U87MG and U251 cells was tested by CCK-8 assay. C) The cell colony-forming ability of U87MG and $\mathrm{U} 251$ cells was detected using colony formation assay. ${ }^{*} p<0.05,{ }^{* *} p<0.01$ vs. Control, si-NC or Vector groups. 


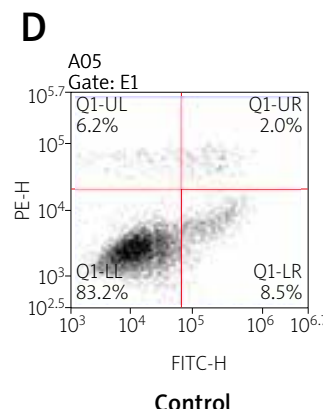

Control

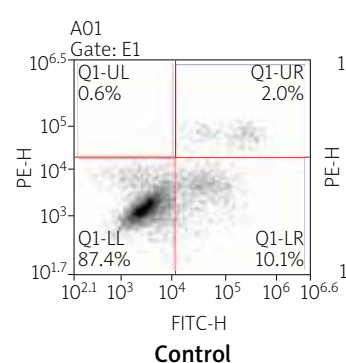

Control

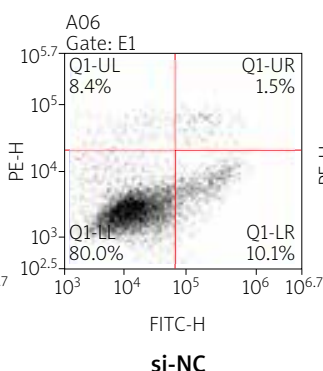

si-NC

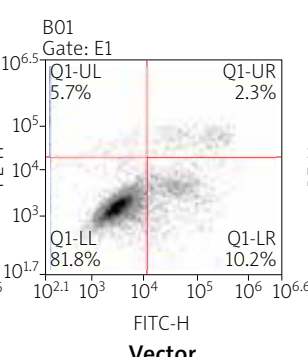

Vector
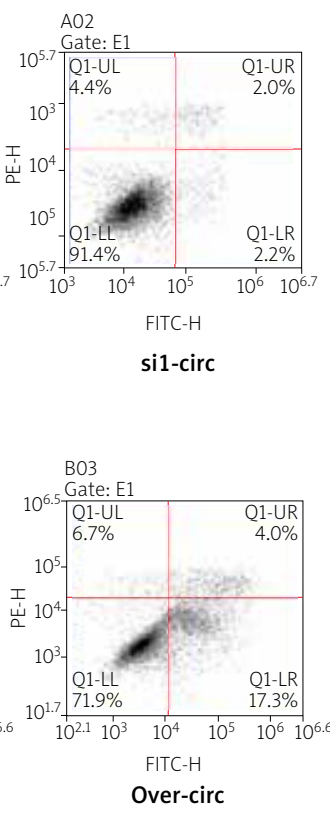

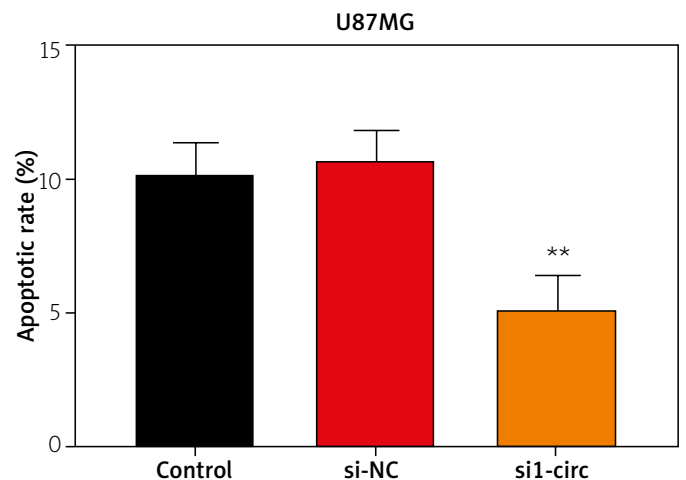

U251

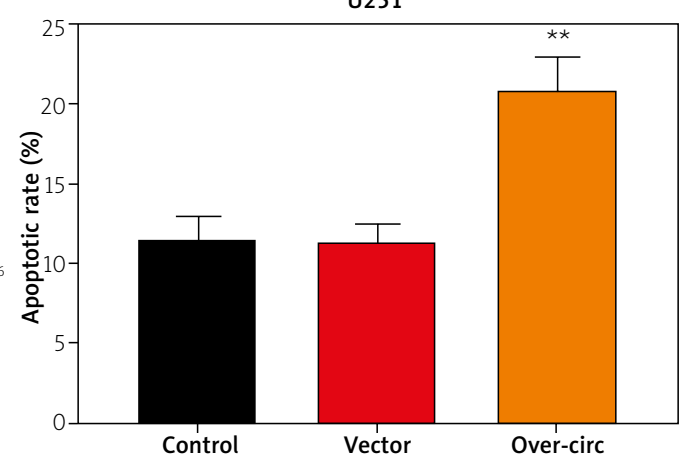

Fig. 2. Cont. D) Cell apoptosis of U87MG and U251 cells was detected using flow cytometer analysis. ${ }^{* *} p<0.01$ vs. Control, si-NC or Vector groups.

property. According to Figure 3C, the overexpressed hsa_circ_0001017 reduced N-cadherin and Vimentin expression and raised E-cadherin expression in U251 cells $(p<0.01)$. Meanwhile the opposite results were observed due to the knockdown of hsa_circ_0001017 of U87MG cells.

\section{Hsa_circ_0001017 sponges hsa-let-7g-3p in glioma}

Many studies showed that circRNAs functioned as miRNA sponges. Next, DGEs and RegRNA2.0 were used to predict miRNAs, and then the intersection of DGEs and RegRNA2.0 identified 12 miRNAs likely to be sponged by hsa_circ_0001017 (Fig. 4A). After overexpressing hsa_circ_0001017, hsa-let-7g-3p expression presented the most downregulated miRNA ( $p<0.01$, Fig. 4B). Therefore, hsalet-7g-3p was selected for further analysis. The Cancer Genome Atlas (TCGA) database showed that hsa-let-7g-3p expression was increased in patients with recurrent glioma compared with glioma patients with non-recurrent glioma, but the difference was not statistically significant ( $p=0.052$, Fig. $4 C$ ). Nota- bly, the survival analysis using the TCGA database showed that high hsa-let-7g-3p expression in lowgrade glioma predicted poor survival $(p=0.017$, Fig. 4D). A series of experiments were conducted to explore whether hsa_circ_0001017 could sponge hsa-let-7g-3p. First, the qRT-PCR assay showed that hsa-let-7g-3p expression in glioma (tumour) tissues was increased ( $p<0.01$, Fig. 4E), which was opposite to hsa_circ_0001017 expression. As shown in Figure 4F, hsa_circ_0001017 and hsa-let-7g-3p could be efficiently pulled down by anti-Ago2 in U251 cells $(p<0.01)$. The potential binding sites of hsalet-7g-3p within the sequence of hsa_circ_0001017 were shown in Figure 4G. The luciferase activity was decreased in U251 cells co-transfected with hsa-let7g-3p mimics and circRNA-0001017-WT, but with no statistically significant differences in other groups (Fig. 4H).

\section{NDST3 is the target gene of hsa-let-7g-3p in glioma}

Three miRNA target gene databases (miRDB, TargetScan, and microRNA.org) were used to seek 
A
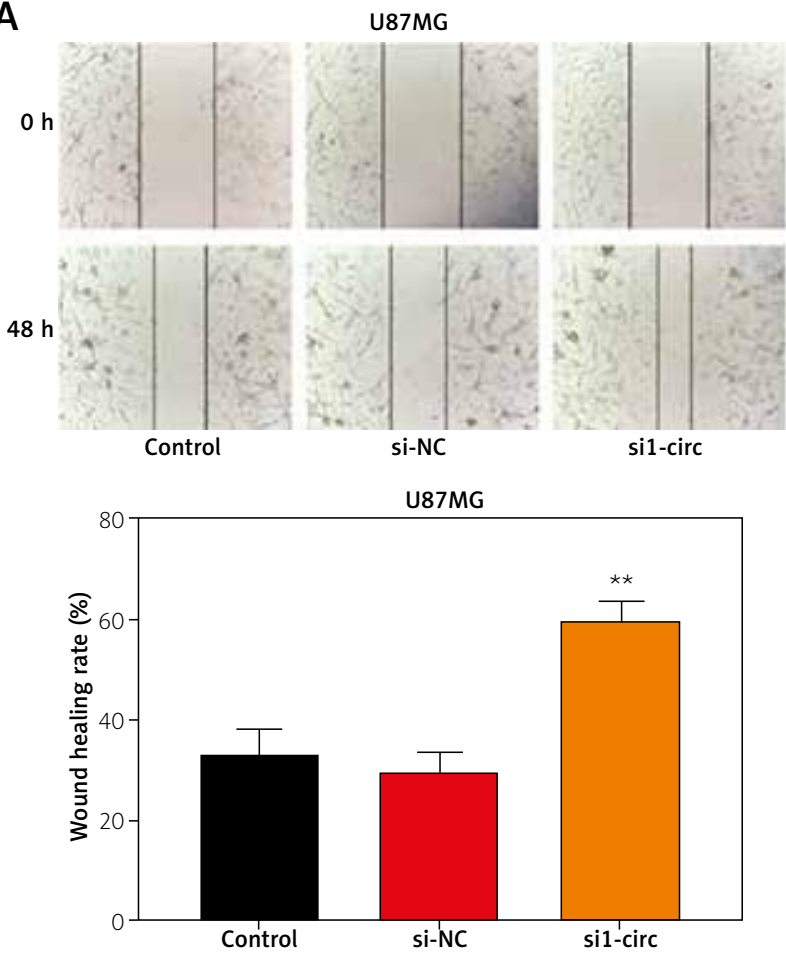
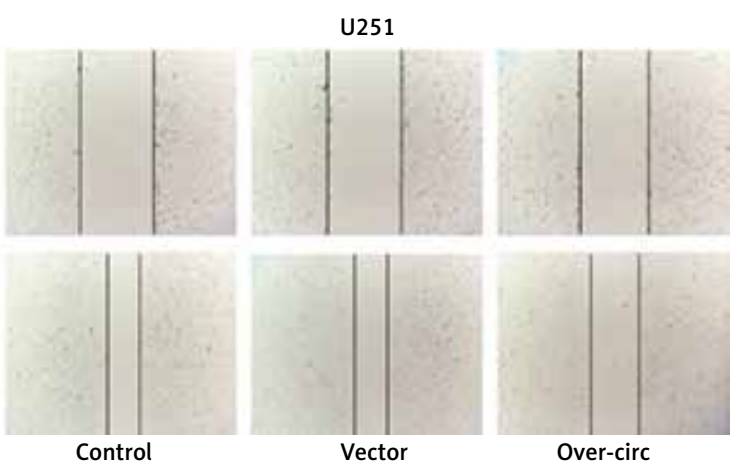

U251

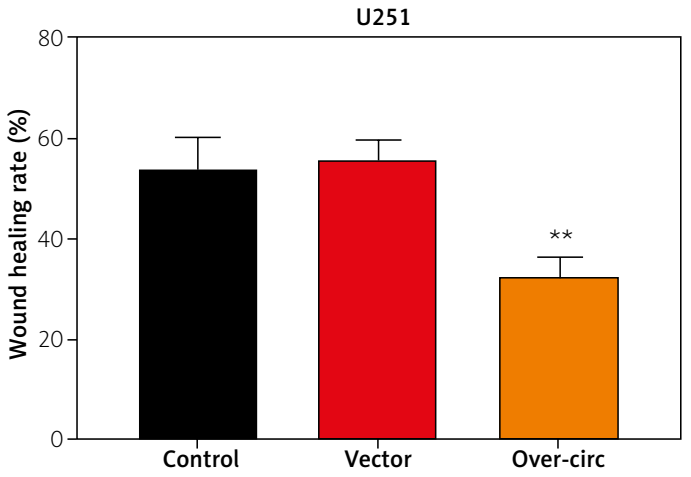

B U87MG U251

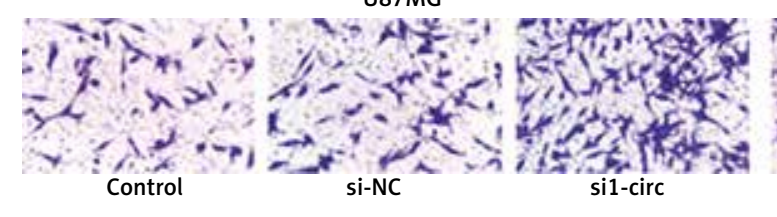

U87MG
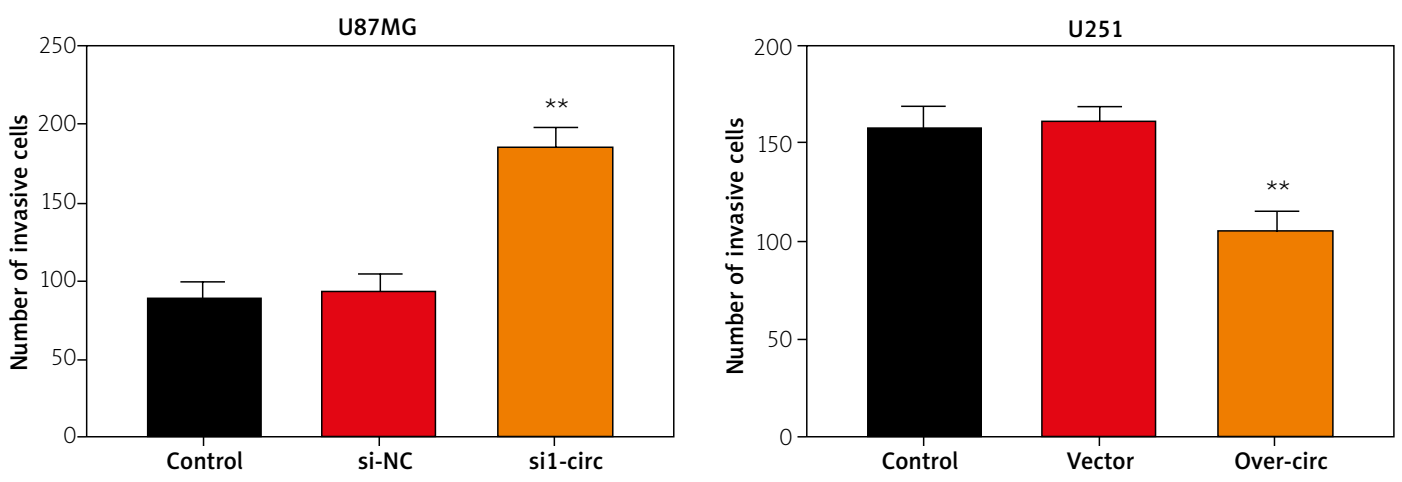

Fig. 3. Glioma cell migration, invasion, and EMT were inhibited by hsa_circ_0001017. A) The cell migration of U87MG and U251 cells was detected using wound healing assay. B) Transwell assay was performed to detect cell invasion of U87MG and U251 cells. ${ }^{* *} p<0.01$ vs. Control, si-NC or Vector groups.

the potential targets of hsa-let-7g-3p so as to illustrate the mechanism of hsa_circ_0001017 as a ceRNA. A total of four miRNAs (MAP4K3, NDST3, LIN28B, and $F N D C 3 A$ ) were identified in both databases (Fig. 5A). Next, the expression of MAP4K3, NDST3, LIN28B, and FNDC3A in glioma tissues was analysed using Gene Expression Profiling Interactive Analysis 2 (GEPIA2). As shown in Figure 5B, NDST3 and LIN28B expression in glioma tissues was decreased, and the difference in NDST3 expression was statistically significant $(p<0.05)$. Therefore, NDST3 was selected for further analysis. Moreover, the TCGA 
C
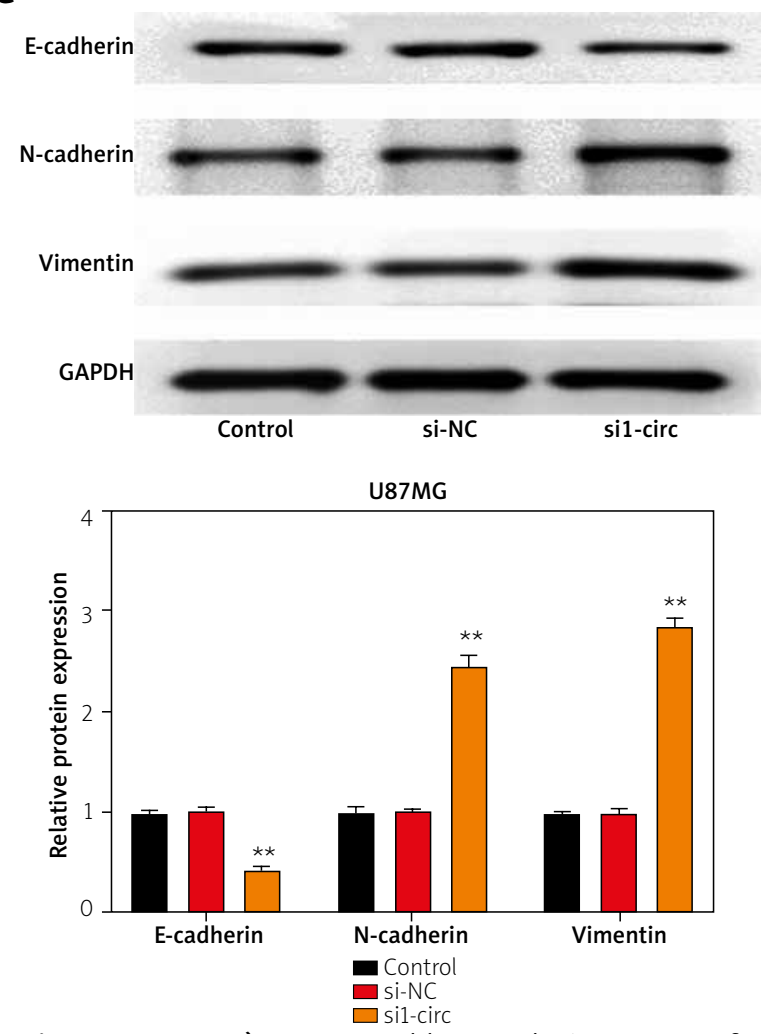

U251
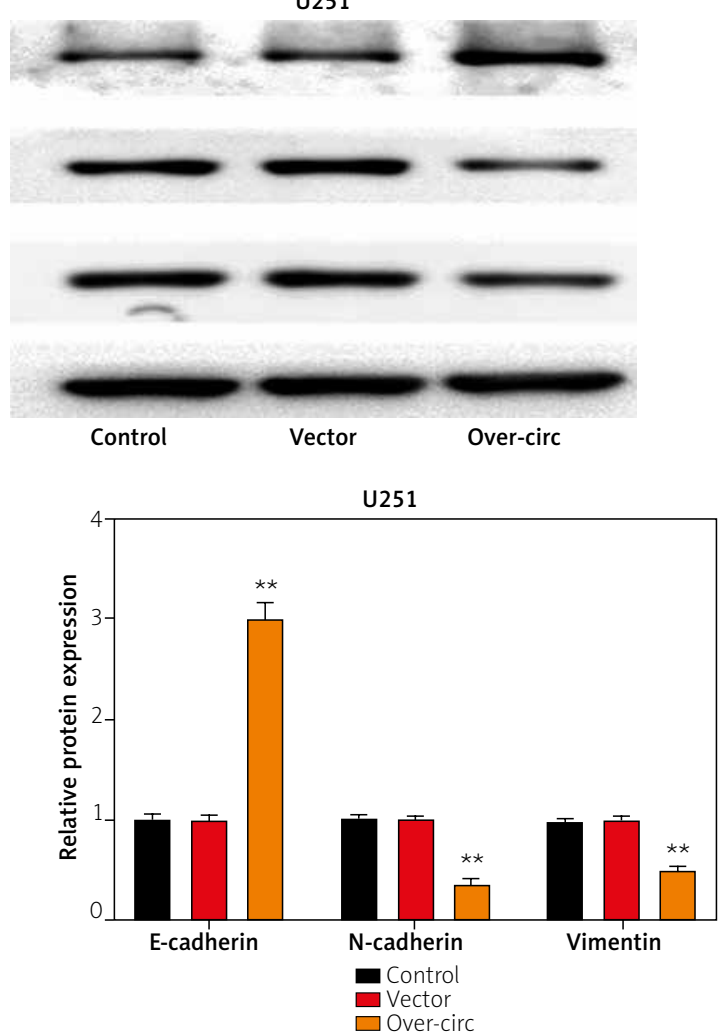

Fig. 3. Cont. C) Western blot analysis was performed to measure EMT-related proteins expression. ${ }^{* *} p<0.01$ vs. Control, si-NC or Vector groups.

database indicated that NDST3 expression was decreased in glioma (Fig. 5C) and high-grade glioma (Fig. 5D), and low NDST3 expression predicted poor survival ( $p=0.0076$, Fig. $5 \mathrm{E}$ ). As shown in Figure $5 \mathrm{~F}$, NDST3 mRNA expression in glioma (tumour) tissues was decreased $(p<0.01)$. The RIP assay found that NDST3 and hsa-let-7g-3p could be efficiently pulled down by anti-Ago2 in U251 cells ( $p<0.01$, Fig. 5G). Figure $5 \mathrm{H}$ displayed the potential binding site of hsalet-7g-3p within NDST3. The luciferase activity was decreased in U251 cells co-transfected with hsa-let$7 \mathrm{~g}-3 \mathrm{p}$ mimic and NDST3-WT, but with no statistically significant differences in other groups (Fig. 5I).

\section{Hsa circ 0001017 restrains the growth and metastasis and induces glioma cell apoptosis via targeting the hsa-let-7g-3p/ NDST3 axis}

U251 cells were transfected with pcDNA 3.1-circ-0001017, let-7g-3p mimic, pcDNA 3.1-circ-0001017 + let-7g-3p mimic or let-7g-3p mimic + pcDNA3.1-NDST3 to conduct rescue assays so as to understand the interplay between hsa_circ_0001017, hsa-let-7g-3p, and NDST3. As indicated in Figure $6 \mathrm{~A}-\mathrm{C}$, the overexpression of hsa_circ_0001017 reduced hsa-let-7g-3p expression and increased NDST3 expression $(p<0.01)$, and the effect of pcDNA3.1-circ-0001017 on NDST3 was restored by let-7g-3p mimic. According to Figure 6D-F, the tumour-suppressive role of circ_0001017 was reversed after co-transfection with let-7g-3p mimic. Moreover, the overexpression of let-7g-3p increased cell proliferation and invasion and decreased cell apoptosis $(p<0.01)$, while overexpression of let-7g-3p and NDST 3 decreased cell proliferation and invasion, and increased cell apoptosis (Fig. 6D-F).

\section{Discussion}

Previous studies reported that rapid proliferation and infiltrative growth were the main causes of death in patients with glioma $[5,23]$. However, the mechanism underlying glioma growth and progression is not clear. Thus, it is particularly important to reveal the potential mechanisms of molecular-targeted therapy in glioma. Accumulating evidence has suggested that circRNAs are closely related to tumour progres- 
A

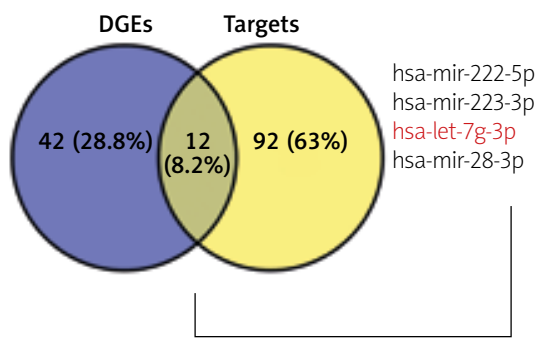

C

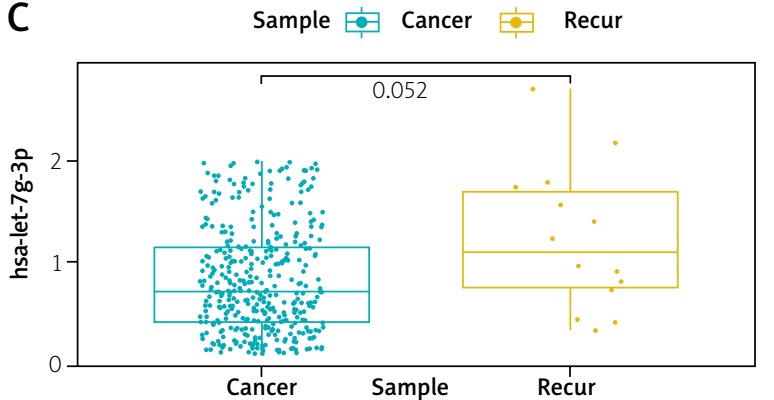

E

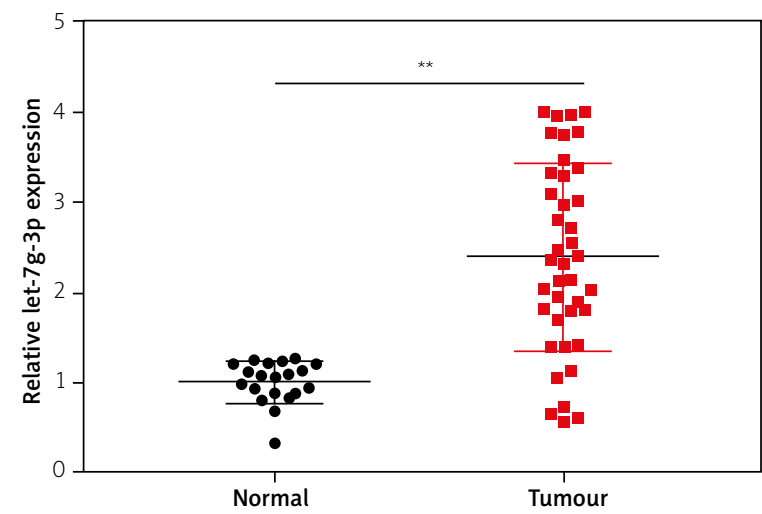

B

hsa-let-7f-5p hsa-mir-27b-5p hsa-mir-22-3p hsa-let-7i-5p hsa-mir-200b-3p hsa-mir-139-5p hsa-mir-125b-1-3p hsa-mir-124-3p

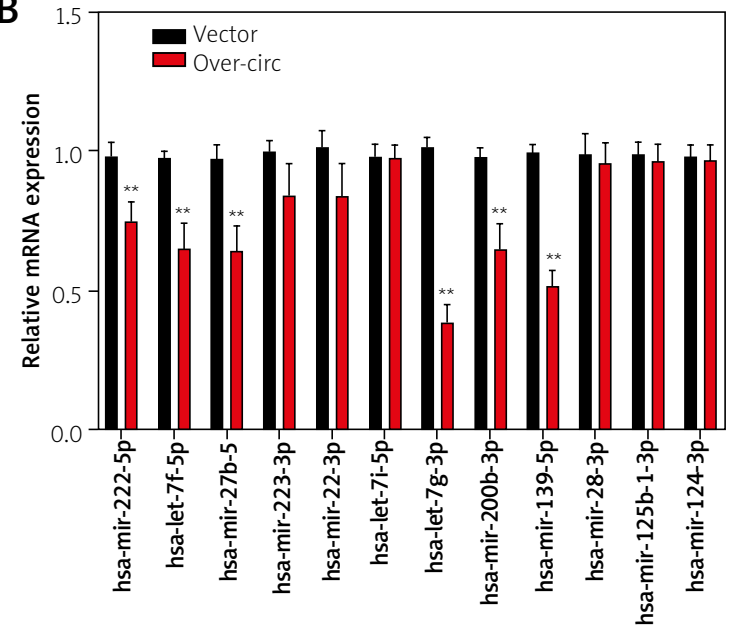

D

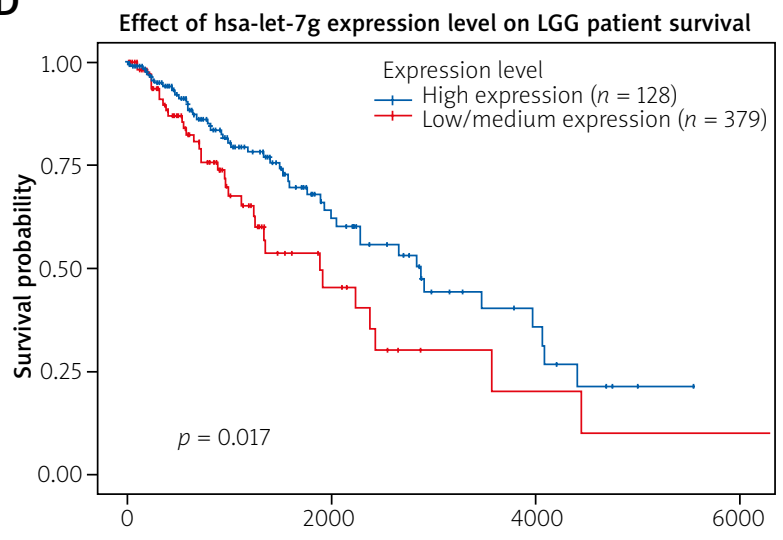

$\mathrm{F}$

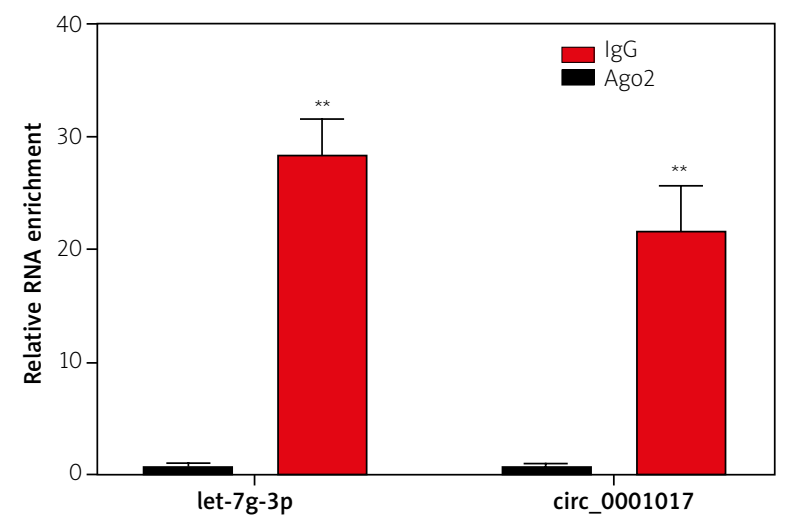

Fig. 4. Hsa_circ_0001017 sponged hsa-let-7g-3p in glioma. A) DGEs and RegRNA2.0 were used to predict miRNAs sponged by hsa_circ_0001017. B) The candidate miRNAs expression in U251 cells with overexpressed hsa_circ_0001017 was measured using qRT-PCR assay; ${ }^{* *} p<0.01$ vs. Vector group. C) Hsa-let-7g-3p expression in recurrent patients with glioma and glioma patients from the Cancer Genome Atlas (TCGA) database. D) Kaplan-Meyer survival curves for two groups of low grade glioma (LGG) patients from TCGA database. E) The hsa-let-7g-3p expression in glioma (tumour) tissues $(n=40)$ and normal tissues $(n=20)$ was measured using qRT-PCR assay; ${ }^{* *} p<0.01$ vs. Normal group. F) After pulling down of Ago2 by RIP assay, hsa_circ_0001017 and hsa-let-7g-3p expression in U251 cells was measured using qRT-PCR assay; ${ }^{* *} p<0.01$ vs. IgG group. 
G

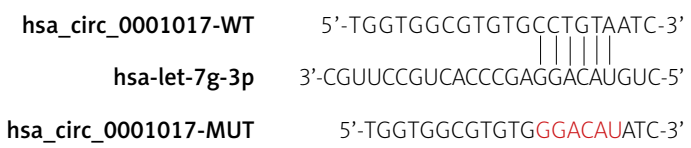

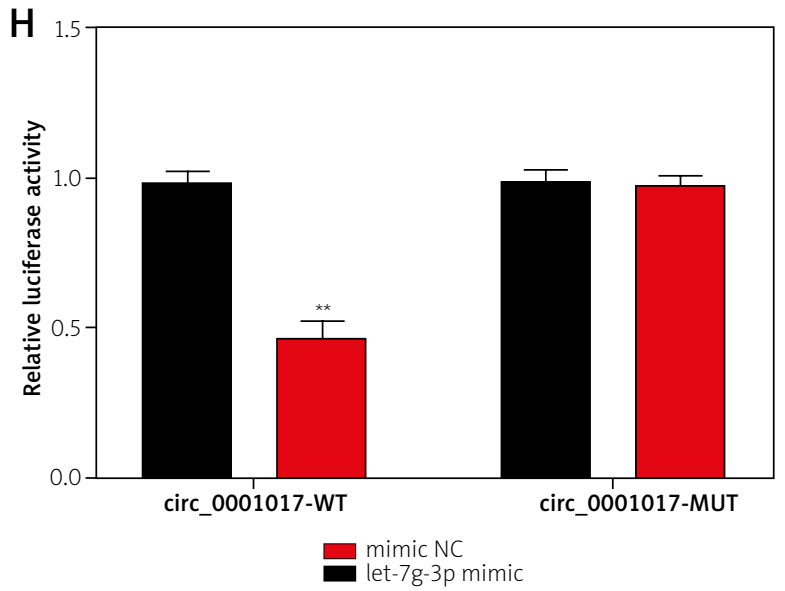

Fig. 4. Cont. G) Potential binding sites of hsa-let-7g-3p within the sequence of hsa circ 0001017 . H) Luciferase reporter assay was performed to test the relative luciferase activity; ${ }^{* *} p<0.01$ vs. mimic NC group.

sion, such as cell proliferation, invasion, and apoptosis $[2,3,13]$. Specifically, many circRNAs have been identified to play a vital role in glioma. Chen et al. [1] found that circPTN promoted proliferation and stemness in glioma. Peng et al. [26] reported that circCPA4 affected the proliferation and metastasis, and served as a prognostic factor for glioma. The present study showed that hsa_circ_0001017 expression decreased in glioma tissues and cells. These findings indicated that hsa_circ_0001017 might participate in the initiation and development of glioma.

The basic biological feature of glioma includes glioma cell proliferation, migration, invasion, and apoptosis [11,33,35]. Therefore, better understanding of the biological features of gliomas may be advantageous to promoting the glioma diagnosis and treatment. The loss/gain of function studies were conducted by transfection with silencing or overexpression plasmid of hsa_circ_0001017 to examine the effects of hsa_circ_0001017 in glioma cells. The overexpressed hsa_circ_0001017 restrained glioma cell proliferation, migration, and invasion, and induced glioma cell apoptosis. However, the knockdown of hsa_circ_0001017 caused the opposite results. Invasion, dissemination, and metastasis are major steps in malignant cancer progression, which are related to EMT $[10,32]$. Thus, it is important to study the EMT process for glioma progression. The expression of EMT-related proteins was examined using western blot analysis to determine whether the influence of hsa_circ_0001017 on glioma migration and invasion resulted from EMT. Experimental evidence indicated that the overex- pressed hsa_circ_0001017 decreased N-cadherin and Vimentin expression and increased E-cadherin expression, whereas the knockdown of hsa circ_0001017 showed contrasting effects. Our findings pointed out that hsa_circ_0001017 might serve as an anti-oncogene in glioma.

CircRNAs regulate tumour progression through sponging miRNA [6]. CiRS-7 was the first circRNA approved as a miRNA sponge. Many studies showed that ciRS-7 sponged miR-7 to regulate gene expression [7,29,34]. In addition to ciRS-7, a lot of other circRNAs have been described to serve as miRNA sponges. CircMMP9 can sponge miR-124 and facilitate glioblastoma multiforme cell tumorigenesis [28]. Circ_001350 regulates glioma cell proliferation, apoptosis, and metastatic properties through sponging miR-1236 [20]. In our study, the bioinformatics analysis was performed to anticipate the target miRNAs of hsa_circ_0001017, revealing the binding sites for hsa-let-7g-3p. López-Aguilar et al. [14] have shown that miR-1303 is upregulated in paediatric astrocytoma, and let-7g-3p has a similar expression pattern to that of miR-1303. We found that hsa-let-7g-3p expression in glioma tissues was higher. In addition, hsa_circ_0001017 could directly interact with hsa-let$7 g-3 p$ and function by sponging hsa-let-7g-3p. These findings suggested that hsa-let-7g-3p was a functional target of hsa_circ_0001017 in glioma.

MiRNAs exert their biological function by binding to 3' UTRs of mRNA. Hence, the online miRNA target bioinformatics analysis was performed to explore possible target genes of hsa-let-7g-3p. NDST3 is expressed in both foetal and adult brains, 
A

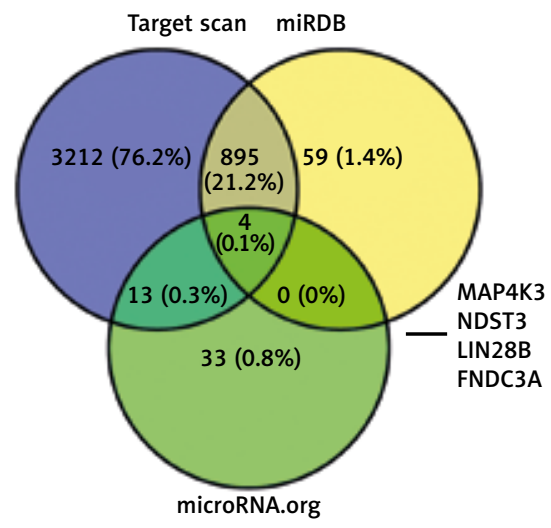

C

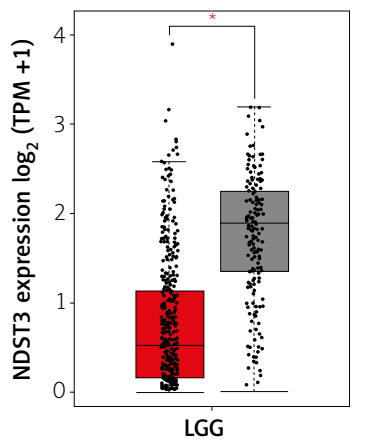

(num $(\mathrm{T})=518, \operatorname{num}(\mathrm{N})=207)$
B

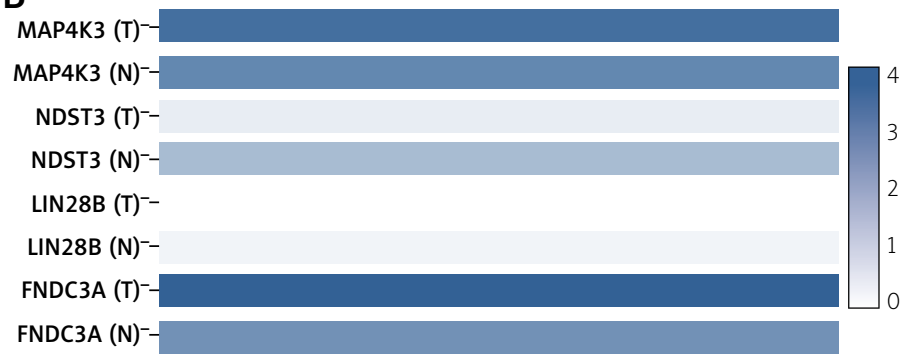

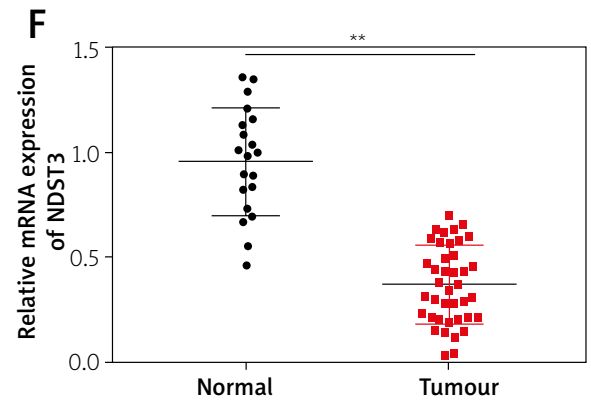

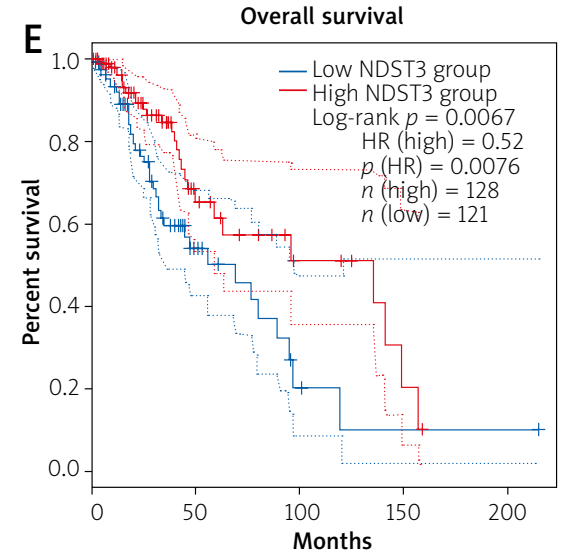

D Expression of NDST3 in LGG based on tumour

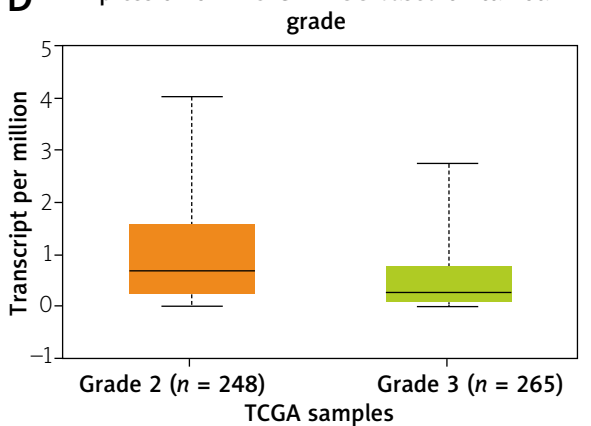

G
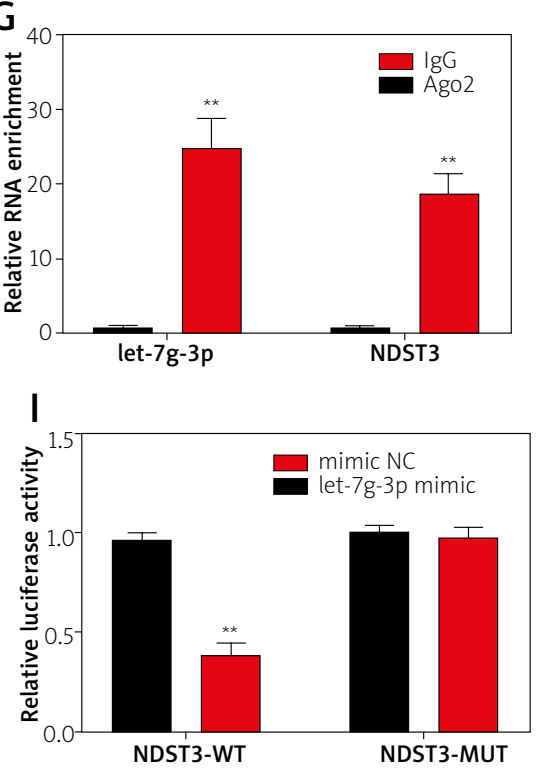

Fig. 5. NDST3 was the target gene of hsa-let-7g-3p in glioma. A) Venn diagram for miRNA target gene databases (miRDB, TargetScan and microRNA.org) of predicting the potential targets of hsa-let-7g-3p. B) The expression of MAP4K3, NDST3, LIN28B and FNDC3A in glioma tissues was analysed using Gene Expression Profiling Interactive Analysis 2 (GEPIA2). C) NDST3 expression in glioma tissues and normal tissues from the Cancer Genome Atlas (TCGA) database. D) NDST3 expression in different grade glioma from TCGA database. E) Kaplan-Meyer survival curves for two groups glioma (LGG patients from TCGA database). F) NDST3 mRNA expression in glioma (tumour) tissues $(n=40)$ and normal tissues $(n=40)$ was tested using qRT-PCR assay; ${ }^{* *} p<0.01$ vs. normal group. G) After pulling down of Ago2 by RIP assay, NDST3 and hsa-let-7g-3p expression was tested in U251 cells using qRT-PCR assay; ${ }^{* *} p<0.01$ vs. IgG group. H) The potential binding site of hsa-let-7g-3p within NDST3. I) Luciferase reporter assay was performed to test the relative luciferase activity; ${ }^{* *} p<0.01$ vs. mimic NC group. 
A

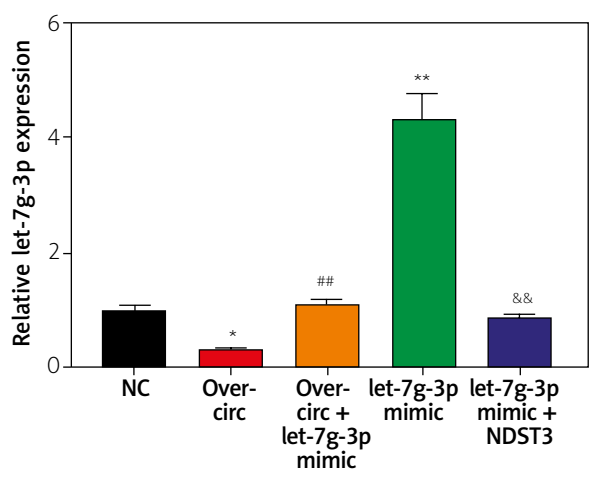

C

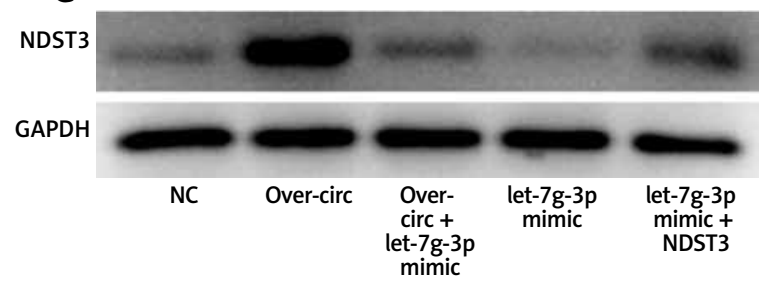

D

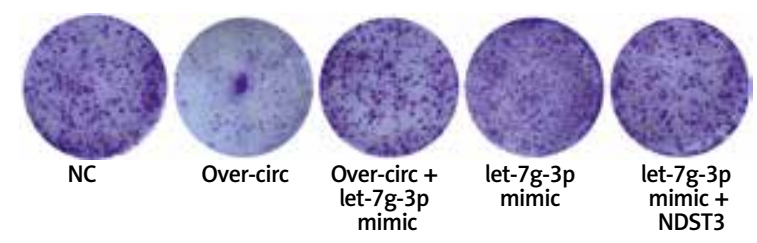

$\mathrm{E}$
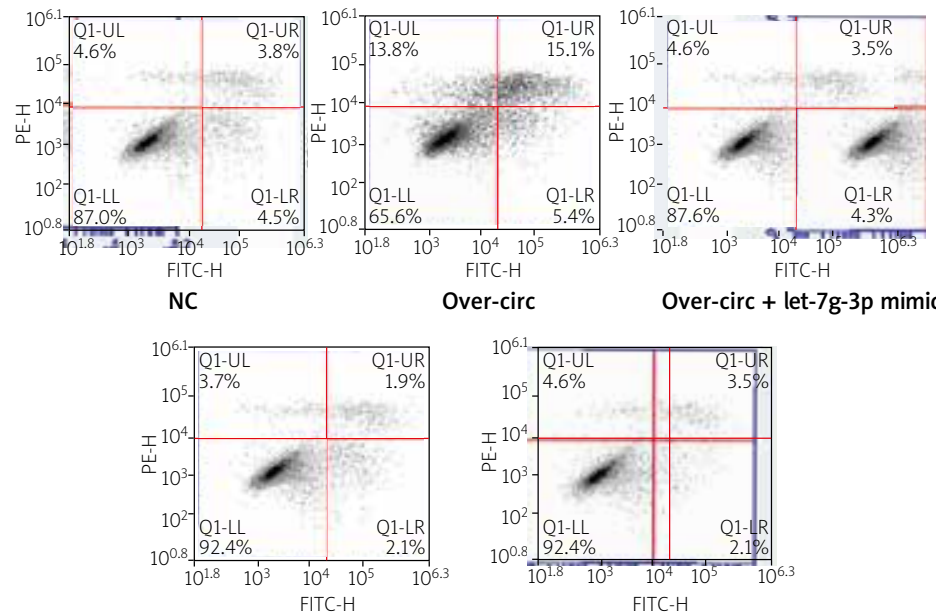

let-7g-3p mimic

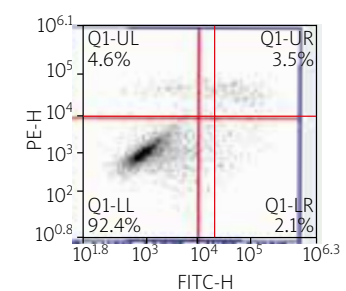

Over-circ + mimic NC
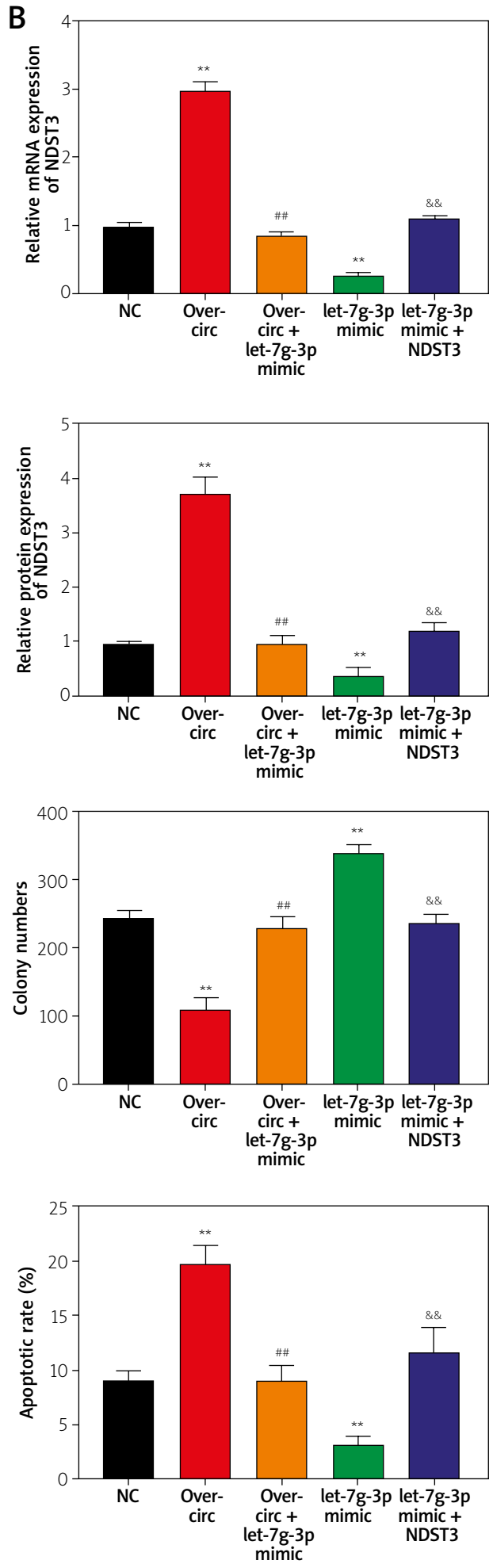

Fig. 6. Hsa_circ_0001017 inhibited the growth and metastasis and promoted cell apoptosis of glioma via targeting the hsa-let-7g-3p/NDST3 axis. A) The hsa-let-7g-3p expression in U251 cells was measured using qRT-PCR assay. B) qRT-PCR assay was performed to detect NDST3 mRNA expression in U251 cells. C) The protein expression of NDST3 was analysed using western blot analysis. D) Cell colony-forming ability of U251 cells was assessed through colony formation assay. E) Apoptosis of U251 cells was detected using flow cytometer analysis. ${ }^{*} p<0.05$ and ${ }^{\star \star} p<0.01$ vs. NC group, ${ }^{\# \#} p<0.01$ vs. Over-circ group, ${ }^{\& \&} p<0.01$ vs. let-7g-3p mimic group. 

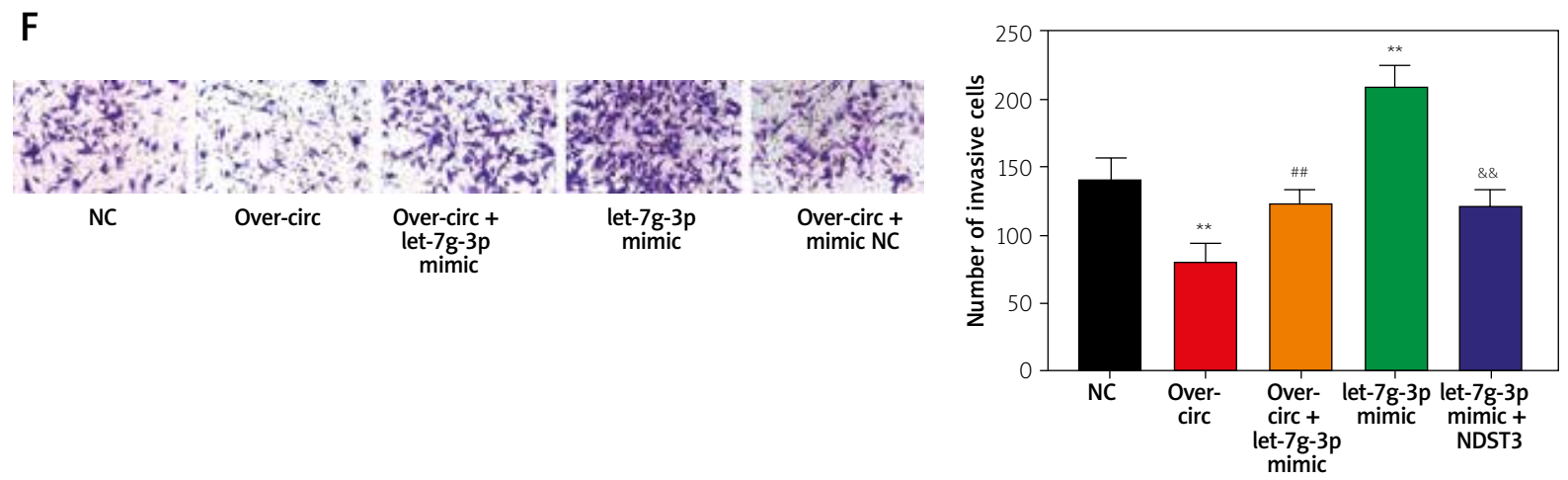

Fig. 6. Cont. F) Transwell assay was performed to detect the invasive ability of U251 cells, ${ }^{* \star} p<0.01$ vs. NC group, ${ }^{\# \#} p<0.01$ vs. Over-circ group, ${ }^{\& \&} p<0.01$ vs. let-7g-3p mimic group.

and it plays a key role in maintaining heparan sulfate metabolism [15,24]. However, NDST3 expression has not yet been reported in glioma. In the present research, NDST3 mRNA expression was decreased in glioma tissues. RIP and luciferase reporter assays showed that NDST3 was the target gene of hsalet-7g-3p in glioma. In addition, further functional studies demonstrated that the overexpression of hsa-let-7g-3p increased proliferation and invasion and decreased apoptosis of glioma cells, while the promotion effect of let-7g-3p overexpression was reversed by the overexpression of NDST3. In addition, the tumour-suppressive effects of circ_0001017 was reversed by overexpression of hsa-let-7g-3p.

\section{Conclusions}

In glioma tissues and cells, hsa_circ_0001017 expression was downregulated. Hsa_circ_0001017 inhibited glioma cell proliferation, migration, and invasion and induced glioma cell apoptosis. Additionally, the tumour-suppressive role of hsa circ_0001017 was related to the hsa-let-7g-3p/ NDST3 axis. Our findings may be conducive to the development of treatment methods for glioma.

\section{Availability of data and material}

The datasets used and analysed during the current study are available from the corresponding author on reasonable request.

\section{Ethics approval}

All enrolled patients did not receive preoperative radiotherapy and chemotherapy before surgery, and they signed written informed consent. The study was approved by the Ethics Committee of the First Affiliated Hospital of Shandong First Medical University.

\section{Disclosure}

The authors report no conflict of interest.

\section{References}

1. Chen J, Chen T, Zhu Y, Li Y, Zhang Y, Wang Y, Li X, Xie X, Wang J, Huang M, Sun X, Ke Y. circPTN sponges miR-145-5p/miR-330$5 p$ to promote proliferation and stemness in glioma. J Exp Clin Cancer Res 2019; 38: 398.

2. Du WW, Fang L, Yang W, Wu N, Awan FM, Yang Z, Yang BB. Induction of tumor apoptosis through a circular RNA enhancing Foxo3 activity. Cell Death Differ 2017; 24: 357-370.

3. Du WW, Yang W, Liu E, Yang Z, Dhaliwal P, Yang BB. Foxo3 circular RNA retards cell cycle progression via forming ternary complexes with p21 and CDK2. Nucleic Acids Res 2016; 44: 28462858.

4. Gittleman H, Boscia A, Ostrom QT, Truitt G, Fritz Y, Kruchko C, Barnholtz-Sloan JS. Survivorship in adults with malignant brain and other central nervous system tumor from 2000-2014. Neuro Oncol 2018; 20: vii6-vii16.

5. Gong W, Wang Z, Wan Y, Shi L, Zhou Y. Downregulation of ABCG2 protein inhibits migration and invasion in U251 glioma stem cells. Neuroreport 2014; 25: 625-632.

6. Han B, Chao J, Yao H. Circular RNA and its mechanisms in disease: from the bench to the clinic. Pharmacol Ther 2018; 187: 31-44.

7. Hansen $\mathrm{TB}$, Jensen $\mathrm{TI}$, Clausen BH, Bramsen JB, Finsen B, Damgaard CK, Kjems J. Natural RNA circles function as efficient microRNA sponges. Nature 2013; 495: 384-388.

8. Hao L, Rong W, Bai L, Cui H, Zhang S, Li Y, Chen D, Meng X. Upregulated circular RNA circ_0007534 indicates an unfavorable prognosis in pancreatic ductal adenocarcinoma and regulates cell proliferation, apoptosis, and invasion by sponging miR-625 and miR-892b. J Cell Biochem 2019; 120: 3780-3789. 
9. He Q, Yan D, Dong W, Bi J, Huang L, Yang M, Huang J, Qin H, Lin T. circRNA circFUT8 upregulates Krüpple-like factor 10 to inhibit the metastasis of bladder cancer via sponging miR-570-3p. Mol Ther Oncolytics 2020; 16: 172-187.

10. Hugo H, Ackland ML, Blick T, Lawrence MG, Clements JA, Williams ED, Thompson EW. Epithelial-mesenchymal and mesenchymal-epithelial transitions in carcinoma progression. J Cell Physiol 2007; 213: 374-383

11. Jakovlevs A, Vanags A, Gardovskis J, Strumfa I. Molecular classification of diffuse gliomas. Pol J Pathol 2019; 70: 246-258.

12. Jeck WR, Sorrentino JA, Wang K, Slevin MK, Burd CE, Liu J, Marzluff WF, Sharpless NE. Circular RNAs are abundant, conserved, and associated with ALU repeats. RNA 2013; 19: 141-157.

13. Kristensen LS, Hansen TB, Venø MT, Kjems J. Circular RNAs in cancer: opportunities and challenges in the field. Oncogene 2018; 37: 555-565.

14. López-Aguilar JE, Velázquez-Flores MA, Simón-Martínez LA, Ávila-Miranda R, Rodríguez-Florido MA, Ruiz-Esparza Garrido R. Circulating microRNAs as biomarkers for pediatric astrocytomas. Arch Med Res 2017; 48: 323-332.

15. Lein ES, Hawrylycz MJ, Ao N, Ayres M, Bensinger A, Bernard A, Boe AF, Boguski MS, Brockway KS, Byrnes EJ, Chen L, Chen TM, Chin MC, Chong J, Crook BE, Czaplinska A, Dang CN, Datta S, Dee NR, Desaki AL, Desta T, Diep E, Dolbeare TA, Donelan MJ, Dong HW, Dougherty JG, Duncan BJ, Ebbert AJ, Eichele G, Estin LK, Faber C, Facer BA, Fields R, Fischer SR, Fliss TP, Frensley C, Gates SN, Glattfelder KJ, Halverson KR, Hart MR, Hohmann JG, Howell MP, Jeung DP, Johnson RA, Karr PT, Kawal R, Kidney JM, Knapik RH, Kuan CL, Lake JH, Laramee AR, Larsen KD, Lau C, Lemon TA, LiangAJ, Liu Y, Luong LT, Michaels J, Morgan JJ, Morgan RJ, Mortrud MT, Mosqueda NF, Ng LL, Ng R, Orta GJ, Overly CC, Pak TH, Parry SE, Pathak SD, Pearson OC, Puchalski RB, Riley ZL, Rockett HR, Rowland SA, Royall J, Ruiz MJ, Sarno NR, Schaffnit K, Shapovalova NV, Sivisay T, Slaughterbeck CR, Smith SC, Smith KA, Smith BI, Sodt AJ, Stewart NN, Stumpf KR, Sunkin SM, Sutram M, Tam A, Teemer CD, Thaller C, Thompson CL, Varnam LR, Visel A, WhitlockRM, WohnoutkaPE, WolkeyCK, WongVY,WoodM, YaylaogluMB, Young RC, Youngstrom BL, YuanXF,Zhang B, Zwingman TA, Jones AR. Genome-wide atlas of gene expression in the adult mouse brain. Nature 2007; 445: 168-176.

16. Li G, Yang H, Han K, Zhu D, Lun P, Zhao Y. A novel circular RNA, hsa_circ_0046701, promotes carcinogenesis by increasing the expression of miR-142-3p target ITGB8 in glioma. Biochem Biophys Res Commun 2018; 498: 254-261.

17. Li P, Chen S, Chen H, Mo X, Li T, Shao Y, Xiao B, Guo J. Using circular RNA as a novel type of biomarker in the screening of gastric cancer. Clin Chim Acta 2015; 444: 132-136.

18. Li T, Shao Y, Fu L, Xie Y, Zhu L, Sun W, Yu R, Xiao B, Guo J. Plasma circular RNA profiling of patients with gastric cancer and their droplet digital RT-PCR detection. J Mol Med (Berl) 2018; 96: 85-96.

19. Li X, Ma N, Zhang Y, Wei H, Zhang H, Pang X, Wu D, Wang D, Yang Z, Zhang S. Circular RNA circNRIP1 promotes migration and invasion in cervical cancer by sponging miR-629-3p and regulating the PTP4A1/ERK1/2 pathway. Cell Death Dis 2020; 11: 399.

20. Liu Y, Ma C, Qin X, Yu H, Shen L, Jin H. Circular RNA circ 001350 regulates glioma cell proliferation, apoptosis, and metastatic properties by acting as a miRNA sponge. J Cell Biochem 2019; 120: 15280-15287

21. Memczak S, Jens M, Elefsinioti A, Torti F, Krueger J, Rybak A, Maier L, Mackowiak SD, Gregersen LH, Munschauer M, Loewer A, Ziebold U, Landthaler M, Kocks C, le Noble F, Rajewsky N. Circular RNAs are a large class of animal RNAs with regulatory potency. Nature 2013; 495: 333-338.

22. Milano MT, Johnson MD, Sul J, Mohile NA, Korones DN, OkunieffP, Walter KA. Primary spinal cord glioma: a surveillance, epidemiology, and end results database study. J Neurooncol 2010; 98: 83-92.

23. Okolie O, Bago JR, Schmid RS, Irvin DM, Bash RE, Miller CR, Hingtgen SD. Reactive astrocytes potentiate tumor aggressiveness in a murine glioma resection and recurrence model. Neuro Oncol 2016; 18: 1622-1633.

24. Pallerla SR, Lawrence R, Lewejohann L, Pan Y, Fischer T, Schlomann U, Zhang X, Esko JD, Grobe K. Altered heparan sulfate structure in mice with deleted NDST3 gene function. J Biol Chem 2008; 283: 16885-16894.

25. Pan Y, Xu T, Liu Y, Li W, Zhang W. Upregulated circular RNA circ_0025033 promotes papillary thyroid cancer cell proliferation and invasion via sponging miR-1231 and miR-1304. Biochem Biophys Res Commun 2019; 510: 334-338.

26. Peng H, Qin C, Zhang C, Su J, Xiao Q, Xiao Y, Xiao K, Liu Q. circCPA4 acts as a prognostic factor and regulates the proliferation and metastasis of glioma. J Cell Mol Med 2019; 23: 6658-6665.

27. Sanger HL, Klotz G, Riesner D, Gross HJ, Kleinschmidt AK. Viroids are single-stranded covalently closed circular RNA molecules existing as highly base-paired rod-like structures. Proc Natl Acad Sci U S A 1976; 73: 3852-3856.

28. Sheng N, Xu YZ, Xi QH, Jiang HY, Wang CY, Zhang Y, Ye Q. Overexpression of KIF2A is suppressed by miR-206 and associated with poor prognosis in ovarian cancer. Cell Physiol Biochem 2018; 50: 810-822.

29. Su C, Han Y, Zhang H, Li Y, Yi L, Wang X, Zhou S, Yu D, Song X, Xiao N, Cao X, Liu Z. CiRS-7 targeting miR-7 modulates the progression of non-small cell lung cancer in a manner dependent on NF-kB signalling. J Cell Mol Med 2018; 22: 3097-3107.

30. Szabo L, Salzman J. Detecting circular RNAs: bioinformatic and experimental challenges. Nat Rev Genet 2016; 17: 679-692.

31. Taylor LP. Diagnosis, treatment, and prognosis of glioma: five new things. Neurology 2010; 75: S28-S32.

32. Valastyan S, Weinberg RA. Tumor metastasis: molecular insights and evolving paradigms. Cell 2011; 147: 275-292.

33. Wang Y, Sui X, Zhao H, Cong L, Li Y, Xin T, Guo M, Hao W. Decreased circular RNA hsa_circ_0001649 predicts unfavorable prognosis in glioma and exerts oncogenic properties in vitro and in vivo. Gene 2018; 676: 117-122.

34. Weng W, Wei Q, Toden S, Yoshida K, Nagasaka T, Fujiwara T, Cai S, Qin H, Ma Y, Goel A. Circular RNA ciRS-7-A promising prognostic biomarker and a potential therapeutic target in colorectal cancer. Clin Cancer Res 2017; 23: 3918-3928.

35. Xu Y, He Y, Xu W, Lu T, Liang W, Jin W. Promotive effects of capillary morphogenetic protein 2 on glioma cell invasion and the molecular mechanism. Folia Neuropathol 2019; 57: 6-15.

36. Xu Y, Yao Y, Zhong X, Leng K, Qin W, Qu L, Cui Y, Jiang X. Downregulated circular RNA hsa_circ_0001649 regulates prolifera- 
tion, migration and invasion in cholangiocarcinoma cells. Biochem Biophys Res Commun 2018; 496: 455-461.

37. Yang M, Li G, Fan L, Zhang G, Xu J, Zhang J. Circular RNA circ_0034642 elevates BATF3 expression and promotes cell proliferation and invasion through miR-1205 in glioma. Biochem Biophys Res Commun 2019; 508: 980-985.

38. Yang Y, Zhang Y, Chen B, Ding L, Mu Z, Li Y. Elevation of circular RNA circ-POSTN facilitates cell growth and invasion by sponging miR-1205 in glioma. J Cell Biochem 2019; 120: 16567-16574.

39. Yu J, Yang M, Zhou B, Luo J, Zhang Z, Zhang W, Yan Z. CircRNA-104718 acts as competing endogenous RNA and promotes hepatocellular carcinoma progression through microRNA-218-5p/ TXNDC5 signaling pathway. Clin Sci 2019; 133: 1487-1503.

40.Zhou Y, Liu F, Xu Q, Wang X. Analysis of the expression profile of Dickkopf-1 gene in human glioma and the association with tumor malignancy. J Exp Clin Cancer Res 2010; 29: 138. 